\title{
FIXED POINTS OF SEMIGROUPS OF LIPSCHITZIAN MAPPINGS DEFINED ON NONCONVEX DOMAINS
}

\author{
KOK-KEONG TAN AND HONG-KUN XU
}

\begin{abstract}
Certain fixed point theorems are established for nonlinear semigroups of Lipschitzian mappings defined on nonconvex domains in Hilbert and Banach spaces. Some known results are thus generalized.
\end{abstract}

\section{INTRODUCTION}

Let $X$ be a Banach space and $C$ be a nonempty subset of $X$. A mapping $T: C \rightarrow C$ is said to be a Lipschitzian mapping if, for each integer $n \geq 1$, there exists a constant $k_{n} \geq 0$ such that $\left\|T^{n} x-T^{n} y\right\| \leq k_{n}\|x-y\| \forall x, y \in C$. A Lipschitzian mapping $T$ is said to be uniformly Lipschitzian if $k_{n}=k$ for all $n \geq 1$, nonexpansive if $k_{n}=1$ for all $n \geq 1$, and asymptotically nonexpansive if $\lim _{n \rightarrow \infty} k_{n}=1$, respectively. Goebel and Kirk [1] initiated in 1973 the study of the fixed point theory for Lipschitzian mappings. They showed that if $X$ is uniformly convex and $C$ is a bounded closed convex subset of $X$, then every uniformly $k$-Lipschitzian mapping $T: C \rightarrow C$ with $k<\gamma$ has a fixed point, where $\gamma>1$ is the unique solution of the equation

$$
\gamma\left[1-\delta_{X}\left(\frac{1}{\gamma}\right)\right]=1,
$$

with $\delta_{X}$ the modulus of convexity of $X$. Since then, much effort has been devoted to the existence theory for fixed points of Lipschitzian mappings in both Hilbert and Banach spaces; see [2], [4], [5], [7], [10], [11], and refereces cited there. Usually the domain $C$ on which $T$ is defined is assumed to be convex. Recently, Ishihara [3] and Takahashi [9] studied in Hilbert spaces the existence theory for fixed points of Lipschitzian mappings which are defined on nonconvex domains. However, their methods do not work outside Hilbert spaces.

1991 Mathematics Subject Classification. 47H10, 47H09 .

Key words and phrases. Fixed point, semigroup, Lipschitzian mapping, nonconvex domain. 
The purpose of the present paper is to investigate the existence theory for the fixed point theory of semigroups of Lipschitzian mappings defined on nonconvex domains in both Hilbert and Banach spaces.

\section{PReliminaries}

Let $G$ be a semitopological semigroup, i.e., $G$ is a semigroup with a Hausdorff topology such that for each $a \in G$, the mappings $t \rightarrow a t$ and $t \rightarrow t a$ from $G$ into itself are continuous. Let $C$ be a nonempty subset of a Banach space $X$. Then a family $\mathcal{F}=\left\{T_{t}: t \in G\right\}$ of self-mappings of $C$ is said to be a Lipschitzian semigroup on $C$ if the following properties are satisfied:

(1) $T_{t s} x=T_{t} T_{s} x$ for all $t, s \in G$ and $x \in C$;

(2) for each $x \in C$, the mapping $t \rightarrow T_{t} x$ is continuous on $G$;

(3) for each $t \in G$, there is a constant $k_{t}>0$ such that $\left\|T_{t} x-T_{t} y\right\| \leq$ $k_{t}\|x-y\| \forall x, y \in C$.

A Lipschitzian semigroup $\mathcal{F}$ is called uniformly $k$-Lipschitzian if $k_{t}=k$ for all $t \in G$ and in particular, nonexpansive if $k_{t}=1$ for all $t \in G$. We shall denote by $F(\mathcal{F})$ the set of common fixed points of $\mathcal{F}=\left\{T_{t}: t \in G\right\}$.

Recall that a semitopological semigroup $G$ is said to be left reversible if any two closed right ideals of $G$ have nonvoid intersection. In this case, $(G, \leq)$ is a directed system when the binary relation " $\leq$ " on $G$ is defined by $a \leq b$ if and only if $\{a\} \cup \overline{a G} \supseteq\{b\} \cup \overline{b G}$. Let $B(G)$ be the Banach space of all bounded real-valued functions on $G$ with the supremum norm and let $X$ be a subspace of $B(G)$ containing constants. Then an element $\mu$ of $X^{*}$, the dual space of $X$, is said to be a mean on $X$ if $\|\mu\|=\mu(1)=1$. It is known that $\mu \in X^{*}$ is a mean on $X$ if and only if the inequalty

$$
\inf \{f(t): t \in G\} \leq \mu(f) \leq \sup \{f(t): t \in G\}
$$

holds for all $f \in X$. For a mean $\mu$ on $X^{*}$ and an element $f \in X$, we use either $\mu_{t}(f(t))$ or $\mu(f)$ to denote the value of $\mu$ at $f$. For each $s \in G$, we define the left transformation $\ell_{s}$ from $B(G)$ into itself by $\left(\ell_{s} f\right)(t)=f(s t)$, $t \in G$, for all $f \in B(G)$. The right transformation $r_{s}$ is defined similarly. Let $X$ be a subspace of $B(G)$ containing constants which is $\ell_{G^{-}}$invariant $\left(r_{G^{-}}\right.$ invariant), i.e., $\ell_{s}(X) \subseteq X\left(r_{s}(X) \subseteq X\right)$ for all $s \in G$. Then a mean $\mu$ on $X$ is said to be left invariant (right invariant) if $\mu(f)=\mu\left(\ell_{s} f\right)\left(\mu(f)=\mu\left(r_{s} f\right)\right)$ for all $f \in X$ and $s \in G$. An invariant mean is a mean that is both left and right invariant.

Let $C(G)$ be the Banach space of all bounded continuous real-valued functions on $G$, let $R U C(G)$ be the space of all bounded right uniformly continuous functions on $G$, i.e., all $f \in C(G)$ for which the mapping $s \rightarrow r_{s} f$ is continuous, and let $A P(G)$ be the space of all $f \in C(G)$ for which $\left\{\ell_{s} f\right.$ : $s \in G\}$ is relatively norm compact. Then $R U C(G)$ is a closed subalgebra of 
$C(G)$ containing constants and both $\ell_{G^{-}}$and $r_{G^{-}}$-invariant; see [7] for details. If $\left\{x_{s}: s \in G\right\}$ is a bounded family of elements of a Banach space $E$, then for all $x \in E$ and $p \geq 1$, the functions $g(s):=\left\|x_{s}-x\right\|^{p}$ and $h(s):=\left\langle x_{s}, x\right\rangle$ (if $E$ is a Hilbert space) are in $R U C(G)$.

\section{The Hilbert Space Setting}

In this section, we prove fixed point theorems for Lipschitzian semigroups defined on nonconvex domains in Hilbert spaces.

Theorem 3.1. Let $C$ be a nonempty subset of a real Hilbert space $H$, let $G$ be a semitopological semigroup such that $R U C(G)$ has a left invariant mean $\mu$, and let $\mathcal{F}=\left\{T_{t}: t \in G\right\}$ be a Lipschitzian semigroup on $C$ such that $\mu_{t}\left(k_{t}^{2}\right)<2$. Suppose that $\left\{T_{t} x: t \in G\right\}$ is bounded and $\cap_{s \in G} \overline{c o}\left\{T_{s t} x\right.$ : $t \in G\}$ is contained in $C$ for all $x \in C$. Then there exists a point $z \in C$ for which $T_{t} z=z$ for all $t \in G$.

Proof. Let $x_{0} \in C$. It is easily seen that the functional $\mu_{t}\left\langle T_{t} x_{0}, x\right\rangle, x \in H$, is a continuous linear functional on $H$. By Riesz's representation theorem, there is a unique element $x_{1} \in H$ satisfying $\mu_{t}\left\langle T_{t} x_{0}, x\right\rangle=\left\langle x_{1}, x\right\rangle, \forall x \in H$. By a routine argument (cf. [3] and [9]) via the separation theorem, we have $x_{1} \in \underset{s \in G}{\cap} \overline{c o}\left\{T_{s t} x_{0}: t \in G\right\}$ and hence by assumption, $x_{1}$ does remain in $C$. Therefore, we can continue the above procedure to obtain a sequence $\left\{x_{n}\right\}_{n=1}^{\infty}$ in $C$ satisfying the following property:

$$
\mu_{t}\left\langle T_{t} x_{n-1}, x\right\rangle=\left\langle x_{n}, x\right\rangle, \quad \forall x \in H, \quad \forall n \geq 1 .
$$

Noting the fact that for all $u, v \in H$, the function $h(t):=\left\|T_{t} u-v\right\|^{2}$ is in $R U C(G)$, it follows from (3.1) that

$$
\begin{aligned}
& \mu_{t}\left\|T_{t} x_{n-1}-x\right\|^{2}=\mu_{t}\left\|\left(T_{t} x_{n-1}-x_{n}\right)+\left(x_{n}-x\right)\right\|^{2}=\mu_{t}\left(\left\|T_{t} x_{n-1}-x_{n}\right\|^{2}+\right. \\
& \left.+\left\|x_{n}-x\right\|^{2}+2\left\langle T_{t} x_{n-1}-x_{n}, x_{n}-x\right\rangle\right)=\mu_{t}\left\|T_{t} x_{n-1}-x_{n}\right\|^{2}+ \\
& +\left\|x_{n}-x\right\|^{2}+2 \mu_{t}\left\langle T_{t} x_{n-1}-x_{n}, x_{n}-x\right\rangle=\mu_{t}\left\|T_{t} x_{n-1}-x_{n}\right\|^{2}+ \\
& +\left\|x_{n}-x\right\|^{2}+2\left\langle x_{n}-x_{n}, x_{n}-x\right\rangle=\mu_{t}\left\|T_{t} x_{n-1}-x_{n}\right\|^{2}+\left\|x_{n}-x\right\|^{2} .
\end{aligned}
$$

This shows that $x_{n}$ is the unique minimizer of the convex function $\mu_{t} \| T_{t} x_{n-1}$ $-x \|^{2}$ over $H$ and in particular, taking $x=T_{s} x_{n}$ and noting that $\mu$ is left invariant, we get

$$
\begin{gathered}
\mu_{t}\left\|T_{t} x_{n-1}-x_{n}\right\|^{2}+\left\|x_{n}-T_{s} x_{n}\right\|^{2}=\mu_{t}\left\|T_{t} x_{n-1}-T_{s} x_{n}\right\|^{2}= \\
=\mu_{t}\left\|T_{s t} x_{n-1}-T_{s} x_{n}\right\|^{2}=\mu_{t}\left\|T_{s} T_{t} x_{n-1}-T_{s} x_{n}\right\|^{2} \leq k_{s}^{2} \mu_{t}\left\|T_{t} x_{n-1}-x_{n}\right\|^{2} .
\end{gathered}
$$

It follows that

$$
\left\|x_{n}-T_{s} x_{n}\right\|^{2} \leq\left(k_{s}^{2}-1\right) \mu_{t}\left\|T_{t} x_{n-1}-x_{n}\right\|^{2} .
$$


Set $A=\mu_{s}\left(k_{s}^{2}-1\right), r_{n}=\mu_{t}\left\|T_{t} x_{n}-x_{n+1}\right\|^{2}$ and $R_{n}=\mu_{t}\left\|T_{t} x_{n}-x_{n}\right\|^{2}$. Then from (3.2) we have

$$
R_{n} \leq A r_{n-1} \leq A R_{n-1} \leq \cdots \leq A^{n} R_{0} .
$$

It then follows from (3.3) that

$$
\begin{aligned}
\left\|x_{n+1}-x_{n}\right\|^{2} & =\mu_{t}\left\|\left(x_{n+1}-T_{t} x_{n}\right)+\left(T_{t} x_{n}-x_{n}\right)\right\|^{2} \leq \\
& \leq 2 \mu_{t}\left(\left\|T_{t} x_{n}-x_{n+1}\right\|^{2}+\left\|T_{t} x_{n}-x_{n}\right\|^{2}\right)= \\
& =2\left(r_{n}+R_{n}\right) \leq 4 R_{n} \leq 4 A^{n} R_{0} .
\end{aligned}
$$

Since $A<1$, we see that $\left\{x_{n}\right\}$ is Cauchy and hence convergent in norm. Let $z$ be the limit of $\left\{x_{n}\right\}$. We claim that $z$ is a common fixed point of $\mathcal{F}$. In fact, for any $s \in G$, since $\mu$ is left invariant, we have

$$
\begin{aligned}
\left\|T_{s} z-z\right\|^{2} & =\mu_{t}\left(\left\|\left(T_{s} z-T_{s t} x_{n}\right)+\left(T_{s t} x_{n}-z\right)\right\|^{2} \leq\right. \\
& \leq 2 \mu_{t}\left(\left\|T_{s t} x_{n}-T_{s} z\right\|^{2}+\left\|T_{s t} x_{n}-z\right\|^{2}\right) \leq \\
& \leq 2\left(k_{s}^{2} \mu_{t}\left\|T_{t} x_{n}-z\right\|^{2}+\mu_{t}\left\|T_{s t} x_{n}-z\right\|^{2}\right)= \\
& =2\left(1+k_{s}^{2}\right) \mu_{t}\left\|T_{t} x_{n}-z\right\|^{2}= \\
& =2\left(1+k_{s}^{2}\right) \mu_{t}\left\|\left(T_{t} x_{n}-x_{n}\right)+\left(x_{n}-z\right)\right\|^{2} \leq \\
& \leq 4\left(1+k_{s}^{2}\right)\left(\mu_{t}\left\|T_{t} x_{n}-x_{n}\right\|^{2}+\left\|x_{n}-z\right\|^{2}\right)= \\
& =4\left(1+k_{s}\right)\left(R_{n}+\left\|x_{n}-z\right\|^{2}\right) \rightarrow 0 \text { as } n \rightarrow \infty .
\end{aligned}
$$

Therefore, $T_{s} z=z$.

Corollary 3.1 (Theorem $3.5[4]$ ). If $\mathcal{F}=\left\{T_{t}: t \in G\right\}$ is a nonexpansive semigroup on a closed convex subset $C$ of a Hilbert space $H$, $R U C(G)$ has a left invariant mean, and there exists an $x \in C$ such that $\left\{T_{s}(x): s \in G\right\}$ is bounded, then $\mathcal{F}$ has a common fixed point in $C$.

Let $X$ be a subspace of $B(G)$ containing constants. Following Mizoguchi and Takahashi [7T], we say that a real valued function on $X$ is a submean on $X$ if the following conditions are fulfilled:

(1) $\mu(f+g) \leq \mu(f)+\mu(g), \quad \forall f, g \in X$;

(2) $\mu(\alpha f) \leq \alpha \mu(f), \quad \forall f \in X, \forall \alpha \geq 0$;

(3) $\forall f, g \in X, f \leq g \Longrightarrow \mu(f) \leq \mu(g)$;

(4) $\mu(c)=c$ for all constants $c$.

Theorem 3.2. Let $H$ be a real Hilbert space, $C$ a nonempty subset of $H, X$ an $\ell_{G}$-invariant subspace of $B(G)$ containing constants that has a left invariant submean $\mu$ on $X$, and $\mathcal{F}=\left\{T_{t}: t \in G\right\}$ a Lipschitzian semigroup on $C$. Suppose that $\left\{T_{t} x: t \in G\right\}$ is bounded for some $x \in C$ and $\cap_{s \in G} \overline{c o}\left\{T_{s t} x: t \in G\right\} \subset C$ for all $x \in C$. Suppose also that for all $u, v \in C$, the function $f$ on $G$ defined by $f(t)=\left\|T_{t} u-v\right\|^{2}$ and the function 
$h$ on $G$ defined by $h(t)=k_{t}^{2}$ belong to $X$ and $\mu_{t}\left(k_{t}^{2}\right)<2$. Then there is a point $z \in C$ such that $T_{t} z=z$ for all $t \in G$.

Proof. Let $x_{0} \in C$ and define the function $r_{0}$ on $H$ by $r_{0}(x)=\mu_{t}\left\|T_{t} x_{0}-x\right\|^{2}$, $x \in H$. Note that $r_{0}$ is well defined since by assumption, the function $t \mapsto\left\|T_{t} x_{0}-x\right\|^{2}$ is in $X$ for every $x \in H$. As $r_{0}$ is strictly convex and continuous and $r_{0}(x) \rightarrow \infty$ as $\|x\| \rightarrow \infty$, there is a unique element $x_{1} \in H$ such that $r_{0}:=r_{0}\left(x_{1}\right)=\inf \left\{r_{0}(x): x \in H\right\}$. We claim that this $x_{1}$ belongs to $\cap_{s \in G} \overline{c o}\left\{T_{s t} x_{0}: t \in G\right\}$ and thus to $C$ by our hypothesis. Indeed, if we denote by $P_{s}$ the nearest point projection of $H$ onto the set $\overline{c o}\left\{T_{s t} x_{0} ; t \in G\right\}$, then, as $P_{s}$ is nonexpansive and $\mu$ is left invariant, we get

$$
\begin{aligned}
r_{0}\left(P_{s} x_{1}\right) & =\mu_{t}\left\|T_{t} x_{0}-P_{s} x_{1}\right\|^{2}=\mu_{t}\left\|T_{s t} x_{0}-P_{s} x_{1}\right\|^{2}= \\
& =\mu_{t}\left\|P_{s} T_{s t} x_{0}-P_{s} x_{1}\right\|^{2} \leq \mu_{t}\left\|T_{s t} x_{0}-x_{1}\right\|^{2}= \\
& =\mu_{t}\left\|T_{t} x_{0}-x_{1}\right\|^{2}=r_{0},
\end{aligned}
$$

which shows that $P_{s} x_{1}$ is also a minimizer of $r_{0}$ and hence by uniqueness, $P_{s} x_{1}=x_{1}$, i.e., $x_{1} \in \cap_{s \in G} \overline{c o}\left\{T_{s t} x_{0}: t \in G\right\}$. This proves the claim. Repeating the above process, we obtain a sequence $\left\{x_{n}\right\}$ in $C$ with the following property:

$$
x_{n} \in \bigcap_{s \in G} \overline{c o}\left\{T_{s t} x_{n-1}: t \in G\right\} \quad \forall n \geq 1
$$

and $x_{n}$ is the unique minimizer over $H$ of the functional $r_{n}(\cdot)$ defined by $r_{n}(x)=\mu_{t}\left\|T_{t} x_{n-1}-x\right\|^{2}, x \in H$. Now by the same argument as in the proof of Theorem 3.1, we conclude that $\left\{x_{n}\right\}$ converges strongly to a common fixed point $z \in C$.

Corollary 3.2 (Theorem 1 [8]). Let $C$ be a closed convex subset of a Hilbert space $H$ and $X$ be an $\ell_{G}$-invariant subspace of $B(G)$ containing constants which has a left invariant submean $\mu$. Let $\mathcal{F}=\left\{T_{t}: t \in G\right\}$ be a Lipschitzian semigroup on $C$ such that $\left\{T_{s} x: s \in G\right\}$ is bounded for some $x \in C$. If for each $u, v \in C$, the function $f(t):=\left\|T_{t} u-v\right\|^{2}$ and the function $g(t):=k_{t}^{2}(t \in G)$ belong to $X$ and $\mu_{s}\left(k_{s}^{2}\right)<2$, then there is $z \in C$ such that $T_{s} z=z$ for all $s \in G$.

We now extend Theorem 3.3 of Lau [4] to a wider class of Lipschitzian semigroups which are defined on nonconvex domains.

Theorem 3.3. Suppose $H$ is a real Hilbert space, $C$ is a nonempty subset of $H$, and $\mathcal{F}=\left\{T_{t}: t \in G\right\}$ is a Lipschitzian semigroup on $C$. Suppose also $A P(G)$ has a left invariant mean $\mu$. If $\mu_{t}\left(k_{t}^{2}\right) \leq 1$ and if there exists an $x \in$ $C$ such that $\left\{T_{t} x: t \in G\right\}$ is relatively compact in norm and $\cap_{s \in G} \overline{c o}\left\{T_{s t} x\right.$ : $t \in G\}$ is contained in $C$, then $\mathcal{F}$ has a common fixed point. 
Proof. Since $\left\{T_{t} x: t \in G\right\}$ is relatively compact, by Lemma 3.1 of Lau [4], for all $y \in H$, the functions $h$ and $g$ defined on $G$ by $h(t)=\left\langle y, T_{t} x\right\rangle$ and $g(t)=\left\|y-T_{t} x\right\|^{2}$ are both in $A P(G)$. So we have a unique $z \in H$ such that $\mu_{t}\left\langle T_{t} x, y\right\rangle=\langle z, y\rangle, \forall y \in H$. As seen before, we have (i) $z \in \cap_{s \in G} \overline{c o}\left\{T_{s t} x\right.$ : $t \in G\}$ and hence $z \in C$ and (ii) $\mu_{t}\left\|T_{t} x-y\right\|^{2}=\mu_{t}\left\|T_{t} x-z\right\|^{2}+\|y-z\|^{2}$ $\forall y \in H$. In particular, we have for all $s \in G$,

$$
\mu_{t}\left\|T_{t} x-T_{s} z\right\|^{2}=\mu_{t}\left\|T_{t} x-z\right\|^{2}+\left\|T_{s} z-z\right\|^{2} .
$$

Noting that

$\mu_{t}\left\|T_{t} x-T_{s} z\right\|^{2}=\mu_{t}\left\|T_{s t} x-T_{s} z\right\|^{2}=\mu_{t}\left\|T_{s} T_{t} x-T_{s} z\right\|^{2} \leq k_{s}^{2} \mu_{t}\left\|T_{t} x-z\right\|^{2}$,

we get for all $s \in G,\left\|T_{s} z-z\right\|^{2} \leq\left(k_{s}^{2}-1\right) \mu_{t}\left\|T_{t} x-z\right\|^{2}$. Hence

$$
\mu_{s}\left\|T_{s} z-z\right\|^{2} \leq\left(\mu_{s}\left(k_{s}^{2}-1\right)\right) \mu_{t}\left\|T_{t} x-z\right\|^{2} \leq 0
$$

for $\mu_{s}\left(k_{s}^{2}\right) \leq 1$; namely, $\mu_{s}\left\|T_{s} z-z\right\|^{2}=0$. Now for $a \in G$, we have

$$
\begin{aligned}
\left\|T_{a} z-z\right\|^{2} & =\mu_{s}\left\|\left(T_{a} z-T_{s} z\right)+\left(T_{s} z-z\right)\right\|^{2} \leq 2 \mu_{s}\left(\left\|T_{a} z-T_{s} z\right\|^{2}+\right. \\
& \left.+\left\|T_{s} z-z\right\|^{2}\right)=2\left(\mu_{s}\left\|T_{s} z-T_{a} z\right\|^{2}+\mu_{s}\left\|T_{s} z-z\right\|^{2}\right)= \\
& =2 \mu_{s}\left\|T_{a s} z-T_{a} z\right\|^{2}=2 \mu\left\|T_{a} T_{s} z-T_{a} z\right\|^{2} \leq \\
& \leq 2 k_{a}^{2} \mu_{s}\left\|T_{s} z-z\right\|^{2}=0 .
\end{aligned}
$$

Therefore $T_{a} z=z$.

Corollary 3.3 (Theorem 3.2 [4]). If $C$ is a closed convex subset of a Hilbert space $H, \mathcal{F}=\left\{T_{t}: t \in G\right\}$ is a nonexpansive semigroup on $C$, $A P(G)$ has a left invariant mean, and $x \in C$ such that $\left\{T_{s} x: s \in G\right\}$ is relatively compact, then $C$ contains a common fixed point for $\mathcal{F}$.

By the same proof as in Theorem 3.1, we get immediately the following result.

Theorem 3.4. Suppose $H$ and $C$ are as in Theorem 3.3 , suppose $A P(G)$ has a left invariant mean $\mu$, and suppose $\mathcal{F}=\left\{T_{t}: t \in G\right\}$ is a Lipschitzian semigroup on $C$ such that $\mu_{t}\left(k_{t}^{2}\right)<2$. If, for every $x \in C,\left\{T_{t} x: t \in G\right\}$ is relatively compact in norm and $\cap_{s \in G} \overline{c o}\left\{T_{s t} x: t \in G\right\}$ is contained in $C$, then $\mathcal{F}$ has a common fixed point. 


\section{The Banach Space Setting}

In this section we study the existence of fixed points for Lipschitzian mappings defined on nonconvex domains in Banach spaces. So suppose $C$ is a nonempty subset of a Banach space $X$ and $\mathcal{F}=\left\{T_{t}: t \in G\right\}$ is a Lipschitzian semigroup defined on $C$. (Here $G$ is as in Section 3 a semitopological semigroup.) We will employ the following condition introduced by Goebel, Kirk, and Thele in [2]: A nonempty subset $E$ of $C$ is said to satisfy the property

(P): For every $x \in E$ and $\varepsilon>0$, there exists $s \in G$ such that $\operatorname{dist}\left(T_{t} x, E\right)<\varepsilon$ for all $t \geq s$.

Recall that the modulus of convexity of a Banach space $X$ is defined as the function

$$
\delta_{X}(\varepsilon)=\inf \left\{1-\frac{1}{2}\|x+y\|: x, y \in B_{X} \text { with }\|x-y\| \geq \varepsilon\right\}, \quad 0 \leq \varepsilon \leq 2,
$$

where $B_{X}$ is the closed unit ball of $X$. A Banach space $X$ is said to be uniformly convex if $\delta_{X}(\varepsilon)>0$ for all $0<\varepsilon \leq 2$. It is said to be $p$-uniformly convex for some $p \geq 2$ if there exists a constant $d>0$ such that $\delta_{X}(\varepsilon) \geq d \varepsilon^{p}$ for $0 \leq \varepsilon \leq 2$. It is known that a Hilbert space is 2-uniformly convex and an $L^{p}(1<p<\infty)$ space is $\max (2, p)$-uniformly convex. We shall need the following characterization of a $p$-uniformly convex Banach space.

Proposition ([cf. [12]). Given a number $p \geq 2$. A Banach space $X$ is p-uniformly convex if and only if there exists a constant $d=d_{p}>0$ such that

$$
\|\lambda x+(1-\lambda) y\|^{p} \leq \lambda\|x\|^{p}+(1-\lambda)\|y\|^{p}-d W_{p}(\lambda)\|x-y\|^{p}
$$

for all $x, y \in X$ and $0 \leq \lambda \leq 1$, where $W_{p}(\lambda)=\lambda^{p}(1-\lambda)+\lambda(1-\lambda)^{p}$.

Theorem 4.1. Suppose $X$ is a p-uniformly convex Banach space for some $p \geq 2, C$ is a nonempty subset of $X, G$ is a semitopological semigroup that is left reversible, and $\mathcal{F}=\left\{T_{t}: t \in G\right\}$ is a uniformly $k$-Lipschitzian semigroup on $C$ with $k<(1+d)^{\frac{1}{p}}$, $d$ being the constant appearing in (4.1). Suppose also there exist an $\bar{x} \in C$ such that $\left\{T_{t} \bar{x}: t \in G\right\}$ is bounded and a nonempty bounded closed convex subset $E$ of $C$ with Property $(P)$. Then there exists a point $z \in E$ such that $T_{t} z=z$ for all $t \in G$.

Proof. Since $\left\{T_{t} \bar{x}: t \in G\right\}$ is bounded, it is easily seen that for all $x \in C$, $\left\{T_{t} x: t \in G\right\}$ is bounded. Now choose any $x_{0} \in E$ and define a functional $f$ on $E$ by $f(x)=\inf _{s} \sup _{t \geq s}\left\|T_{t} x_{0}-x\right\|^{p}, x \in E$. By Lemma 3 of [11], we have a unique $x_{1} \in E$ such that $f\left(x_{1}\right) \leq f(x)-d\left\|x-x_{1}\right\|^{p}, \forall x \in E$. 
Continuing this procedure, we construct a sequence $\left\{x_{n}\right\}_{n=1}^{\infty}$ in $E$ such that for every integer $n \geq 1$,

$$
\begin{gathered}
\inf _{s} \sup _{t \geq s}\left\|T_{t} x_{n-1}-x_{n}\right\|^{p} \leq \inf _{s} \sup _{t \geq s}\left\|T_{t} x_{n-1}-x\right\|^{p}- \\
-d\left\|x-x_{n}\right\|^{p}, \quad \forall x \in E .
\end{gathered}
$$

Now by Property (P), we can find for each $n \geq 1$ an $a_{n} \in G$ such that

$$
\operatorname{dist}\left(T_{a} x_{n}, E\right)<A^{n} \quad \text { for all } a \geq a_{n},
$$

where $A=\frac{k^{p}-1}{d}<1$. For each fixed $a \geq a_{n}$, from (4.3) we can thus find a $y_{n} \in E$ such that

$$
\left\|T_{a} x_{n}-y_{n}\right\|<A^{n} .
$$

Using the mean value theorem, it is easy to see that for all $x, y \in X$,

$\left|\|y\|^{p}-\|x\|^{p}\right| \leq p[\max \{\|x\|,\|y\|\}]^{p-1}|\|y\|-\|x\|| \leq p(\|x\|+\|y\|)^{p-1}\|y-x\|$.

Since all the involved sequences are bounded, we can find a constant $M$ big enough so that for all $t, a \in G$ and $n \geq 1$,

$$
p\left(\left\|T_{t} x_{n-1}-y_{n}\right\|+\left\|T_{t} x_{n-1}-T_{a} x_{n}\right\|\right)^{p-1} \leq M / 2
$$

and

$$
p\left(\left\|T_{a} x_{n}-x_{n}\right\|+\left\|y_{n}-x_{n}\right\|\right)^{p-1} \leq M / 2 .
$$

It thus follows from (4.2) and (4.4) that

$$
\begin{aligned}
& \inf _{s} \sup _{t \geq s}\left\|T_{t} x_{n-1}-x_{n}\right\|^{p} \leq \inf _{s} \sup _{t \geq s}\left\|T_{t} x_{n-1}-y_{n}\right\|^{p}-d\left\|y_{n}-x_{n}\right\|^{p} \leq \\
& \leq \inf _{s} \sup _{t \geq s}\left\|T_{t} x_{n-1}-T_{a} x_{n}\right\|^{p}-d\left\|T_{a} x_{n}-x_{n}\right\|^{p}+\sup _{t}\left(\| T_{t} x_{n-1}-\right. \\
& \left.-y_{n}\left\|^{p}-\right\| T_{t} x_{n-1}-T_{a} x_{n} \|^{p}\right)+d\left(\left\|T_{a} x_{n}-x_{n}\right\|^{p}-\left\|y_{n}-x_{n}\right\|^{p}\right) \leq \\
& \leq \inf _{s} \sup _{t \geq s}\left\|T_{t} x_{n-1}-T_{a} x_{n}\right\|^{p}-d\left\|T_{a} x_{n}-x_{n}\right\|^{p}+M\left\|T_{a} x_{n}-y_{n}\right\| \leq \\
& \leq \inf _{s} \sup _{t \geq s}\left\|T_{t} x_{n-1}-T_{a} x_{n}\right\|^{p}-d\left\|T_{a} x_{n}-x_{n}\right\|^{p}+M A^{n} \leq \\
& \leq \inf _{s} \sup _{t \geq s}\left\|T_{a t} x_{n-1}-T_{a} x_{n}\right\|^{p}-d\left\|T_{a} x_{n}-x_{n}\right\|^{p}+M A^{n} \leq \\
& \leq k_{a}^{p} \inf _{s} \sup _{t \geq s}\left\|T_{t} x_{n-1}-x_{n}\right\|^{p}-d\left\|T_{a} x_{n}-x_{n}\right\|^{p}+M A^{n} .
\end{aligned}
$$

Hence for all $a \geq a_{n}$,

$$
d\left\|T_{a} x_{n}-x_{n}\right\|^{p} \leq\left(k^{p}-1\right) \inf _{s} \sup _{t \geq s}\left\|T_{t} x_{n-1}-x_{n}\right\|^{p}+M A^{n},
$$


which results in the conclusion

$$
\inf _{s} \sup _{t \geq s}\left\|T_{t} x_{n}-x_{n}\right\|^{p} \leq A \inf _{s} \sup _{t \geq s}\left\|T_{t} x_{n-1}-x_{n}\right\|^{p}+M^{\prime} A^{n},
$$

where $M^{\prime}=M / d$. Write $R_{n}=\inf _{s} \sup _{t>s}\left\|T_{t} x_{n}-x_{n}\right\|^{p}$ and $r_{n}=$ $\inf _{s} \sup _{t \geq s}\left\|T_{t} x_{n}-x_{n+1}\right\|^{p}$. Then $r_{n} \leq R_{n}$ by (4.2) and

$$
\begin{aligned}
R_{n} & \leq A r_{n-1}+M^{\prime} A^{n} \leq A R_{n-1}+M^{\prime} A^{n} \leq A\left(A R_{n-2}+M^{\prime} A^{n-1}\right)+ \\
& +M^{\prime} A^{n}=A^{2} R_{n-2}+2 M^{\prime} A^{n} \leq \cdots \leq\left(R_{0}+n M^{\prime}\right) A^{n} .
\end{aligned}
$$

Therefore,

$$
\begin{aligned}
\left\|x_{n}-x_{n-1}\right\|^{p} & =\inf _{s} \sup _{t \geq s}\left\|\left(x_{n}-T_{t} x_{n-1}\right)+\left(T_{t} x_{n-1}-x_{n-1}\right)\right\|^{p} \leq \\
& \leq 2^{p-1} \inf _{s} \sup _{t \geq s}\left(\left\|x_{n}-T_{t} x_{n-1}\right\|^{p}+\left\|T_{t} x_{n-1}-x_{n-1}\right\|^{p}\right) \leq \\
& \leq 2^{p-1}\left(r_{n-1}+R_{n-1}\right) \leq 2^{p} R_{n-1} \leq 2^{p}\left(R_{0}+(n-1) M^{\prime}\right) A^{n-1},
\end{aligned}
$$

which shows that $\left\{x_{n}\right\}$ is Cauchy and hence convergent to some $z$ strongly. We now show that this $z$ is a common fixed point of $\mathcal{F}$. In fact, noting that the inequality (cf. [11])

$$
\inf _{s} \sup _{t \geq s}\left\|T_{t} x-y\right\|^{p} \leq \inf _{s} \sup _{t \geq s}\left\|T_{a t} x-y\right\|^{p}
$$

is valid for all $x, y \in C$ and $a \in G$, we have for all $a \in G$,

$$
\begin{aligned}
& \left\|z-T_{a} z\right\|^{p} \leq \inf _{s} \sup _{t \geq s}\left(\left\|z-x_{n}\right\|+\left\|x_{n}-T_{t} x_{n}\right\|+\right. \\
& \left.+\left\|T_{t} x_{n}-T_{a} x_{n}\right\|+\left\|T_{a} x_{n}-T_{a} z\right\|\right)^{p} \leq 4^{p} \inf _{s} \sup _{t \geq s}\left(\left(1+k^{p}\right)\left\|z-x_{n}\right\|^{p}+\right. \\
& \left.+\left\|x_{n}-T_{t} x_{n}\right\|^{p}+\left\|T_{t} x_{n}-T_{a} x_{n}\right\|^{p}\right) \leq 4^{p}\left(\left(1+k^{p}\right)\left\|z-x_{n}\right\|^{p}+R_{n}+\right. \\
& \left.+\inf _{s} \sup _{t \geq s}\left\|T_{a t} x_{n}-T_{a} x_{n}\right\|^{p}\right) \leq 4^{p}\left(1+k^{p}\right)\left(\left\|z-x_{n}\right\|^{p}+R_{n}\right) \rightarrow 0(n \rightarrow \infty) .
\end{aligned}
$$

Hence $T_{a} z=z$.

Theorem 4.2. Let $X$ be a p-uniformly convex Banach space for some $p \geq 2, C$ a nonempty subset of $X, G$ a semitopological semigroup for which $R U C(G)$ has a left invariant mean $\mu$, and $\mathcal{F}=\left\{T_{t}: t \in G\right\}$ a uniformly $k$-Lipschitzian semigroup on $C$ such that $k<(1+d)^{\frac{1}{p}}$ with $d$ being the constant appearing in (4.1). Suppose there is a point $\bar{x} \in C$ for which the orbit $\left\{T_{t} \bar{x}: t \in G\right\}$ is bounded. Suppose also there exists a nonempty bounded closed convex subset $E$ of $C$ with the following property:

$\left(P^{*}\right)$ For every $x \in E$ and $\varepsilon>0$, there exists $s \in G$ such that $\operatorname{dist}\left(T_{s t} x, E\right)$ $<\varepsilon, \forall t \in G$.

Then there exists some $z \in E$ such that $T_{t} z=z$ for all $t \in G$. 
Proof. Choose an arbitrary $x_{0} \in E$. As the function $t \mapsto\left\|T_{t} u-v\right\|^{p}$ is in $R U C(G)$ for any $u \in C$ and $v \in X$, we can inductively construct a sequence $\left\{x_{n}\right\}_{n=1}^{\infty}$ in $E$ in the following way: For each integer $n \geq 1, x_{n} \in E$ is the unique minimizer of the functional $\mu_{t}\left\|T_{t} x_{n-1}-x\right\|^{2}$ over $E$. Then by Lemma 2 of [11], we have

$$
\mu_{t}\left\|T_{t} x_{n-1}-x_{n}\right\|^{p} \leq \mu_{t}\left\|T_{t} x_{n-1}-x\right\|^{p}-d\left\|x-x_{n}\right\|^{p}, \quad \forall x \in E .
$$

Write $r_{n}=\inf \left\{\mu_{t}\left\|T_{t} x_{n}-x\right\|^{p}: x \in E\right\}=\mu_{t}\left\|T_{t} x_{n}-x_{n+1}\right\|^{p}$ and $R_{n}=$ $\mu_{t}\left\|T_{t} x_{n}-x_{n}\right\|^{p}$. Then for a fixed integer $n \geq 1$, condition $\left(P^{*}\right)$ yields an $s_{n} \in G$ such that

$$
\operatorname{dist}\left(T_{s_{n} r} x_{n}, E\right)<A^{n}, \quad \forall r \in G,
$$

where $A=\left(k^{p}-1\right) / d<1$. From (4.6) we can find a $y_{n} \in E$ (depending on $r$ ) such that $\left\|T_{s_{n} r} x_{n}-y_{n}\right\|<A^{n}$. It then follows from (4.5) that

$$
\begin{aligned}
r_{n-1} & \leq \mu_{t}\left\|T_{t} x_{n-1}-y_{n}\right\|^{p}-d\left\|y_{n}-x_{n}\right\|^{p}=\mu_{t} \|\left(T_{t} x_{n-1}-T_{s_{n} r} x_{n}\right)+ \\
& +\left(T_{s_{n} r} x_{n}-y_{n}\right)\left\|^{p}-d\right\|\left(y_{n}-T_{s_{n} r} x_{n}\right)+\left(T_{s_{n} r} x_{n}-x_{n}\right) \|^{p} \leq \\
& \leq \mu_{t}\left\|T_{t} x_{n-1}-T_{s_{n} r} x_{n}\right\|^{p}-d\left\|T_{s_{n} r} x_{n}-x_{n}\right\|+M A^{n}= \\
& =\mu_{t}\left\|T_{s_{n} r t} x_{n-1}-T_{s_{n} r} x_{n}\right\|^{p}-d\left\|T_{s_{n} r} x_{n}-x_{n}\right\|^{p}+M A^{n} \leq \\
& \leq k^{p} \mu_{t}\left\|T_{t} x_{n-1}-x_{n}\right\|^{p}-d\left\|T_{s_{n} r} x_{n}-x_{n}\right\|^{p}+M A^{n},
\end{aligned}
$$

where $M>0$ is some appropriate constant independent of $r \in G$, which can be found similarly to the proof of Theorem 4.2. Hence

$$
\left\|T_{s_{n} r} x_{n}-x_{n}\right\|^{p} \leq \frac{k^{p}-1}{d} r_{n-1}+\frac{M}{d} A^{n}=A r_{n-1}+M^{\prime} A^{n},
$$

where $M^{\prime}=M / d$, and

$$
\begin{aligned}
R_{n} & =\mu_{t}\left\|T_{t} x_{n}-x_{n}\right\|^{p}=\mu_{r}\left\|T_{s_{n} r} x_{n}-x_{n}\right\|^{p} \leq \\
& \leq A r_{n-1}+M^{\prime} A^{n} \leq \cdots \leq\left(R_{0}+n M^{\prime}\right) A^{n} .
\end{aligned}
$$

Therefore, we have

$$
\begin{aligned}
\left\|x_{n}-x_{n-1}\right\|^{p} & \leq \mu_{t}\left\|\left(x_{n}-T_{t} x_{n-1}\right)+\left(T_{t} x_{n-1}-x_{n-1}\right)\right\|^{p} \leq \\
& \leq 2^{p} \mu\left(\left\|x_{n}-T_{t} x_{n-1}\right\|^{p}+\left\|T_{t} x_{n-1}-x_{n-1}\right\|^{p}\right)= \\
& =2^{p}\left(r_{n-1}+R_{n-1}\right) \leq 2^{p+1} R_{n-1} \leq \\
& \leq 2^{p+1}\left(R_{0}+(n-1) M^{\prime}\right) A^{n-1},
\end{aligned}
$$


showing that $\left\{x_{n}\right\}$ is Cauchy and hence strongly convergent. Let $z$ be the limit. Now for all $a \in G$, we have

$$
\begin{aligned}
& \left\|z-T_{a} z\right\|^{p} \leq \mu_{t}\left(\left\|z-x_{n}\right\|+\left\|x_{n}-T_{t} x_{n}\right\|+\left\|T_{t} x_{n}-T_{a} x_{n}\right\|+\right. \\
& \left.+\left\|T_{a} x_{n}-T_{a} z\right\|\right)^{p} \leq 4^{p} \mu_{t}\left(\left\|z-x_{n}\right\|^{p}+\left\|x_{n}-T_{t} x_{n}\right\|^{p}+\left\|T_{t} x_{n}-T_{a} x_{n}\right\|^{p}+\right. \\
& \left.+\left\|T_{a} x_{n}-T_{a} z\right\|^{p}\right) \leq 4^{p}\left(\left(1+k^{p}\right)\left\|x_{n}-z\right\|^{p}+R_{n}+\mu_{t}\left\|T_{a t} x_{n}-T_{a} z_{n}\right\|^{p}\right) \leq \\
& \leq 4^{p}\left(1+k^{p}\right)\left(\left\|x_{n}-z\right\|^{p}+R_{n}\right) \rightarrow 0(n \rightarrow \infty) .
\end{aligned}
$$

Hence $T_{a} z=z$.

In $L^{p}(1<p<\infty)$, we have the following inequalities (cf. [5], [6], [12]):

$$
\|\lambda x+(1-\lambda) y\|^{q} \leq \lambda\|x\|^{q}+(1-\lambda)\|y\|^{q}-d_{p} W_{q}(\lambda)\|x-y\|^{q}
$$

for all $x, y \in L^{p}$ and $\lambda \in[0,1]$, where $q=\max \{2, p\}, W_{q}(\lambda)=\lambda^{q}(1-\lambda)+$ $\lambda(1-\lambda)^{q}$, and

$$
d_{p}= \begin{cases}\frac{\left(1+t_{p}^{p-1}\right)}{\left(1+t_{p}\right)^{p-1}}, & \text { if } 2<p<\infty \\ p-1, & \text { if } 1<p \leq 2 .\end{cases}
$$

$t_{p}$ is the unique solution of the equation $(p-2) t^{p-1}+(p-1) t^{p-2}-1=0$, $t \in(0,1)$. Thus we have the following consequence of Theorems 4.1 and 4.2.

Corollary 4.1. Let $C$ be a nonempty subset of $L^{p}(1<p<\infty), G$ a semitopological semigroup which is left reversible or for which the space $R U C(G)$ has an invariant mean, and $\mathcal{F}=\left\{T_{t}: t \in G\right\}$ a uniformly $k$ Lipschitzian semigroup on $C$ with $k<\sqrt{p}$ if $1<p \leq 2$ or $k<1+\left(1+t_{p}^{p-1}\right)$ $\left.\left(1+t_{p}\right)^{1-p}\right]^{\frac{1}{p}}$ if $2<p<\infty$. Suppose there exists an $x \in C$ such that the orbit $\left\{T_{t} x: t \in G\right\}$ is bounded. Suppose also there exists a nonempty bounded closed convex subset of $C$ which possesses Property $(P)$ in the case where $G$ is left reversible or Property $\left(P^{*}\right)$ in the case $R U C(G)$ has an invariant mean. Then there exists a $z \in E$ such that $T_{s} z=z$ for all $s \in G$.

\section{ACKNOWLEDGEMENT}

The authors would like to thank the anonymous referee for a careful reading of the manuscript and helpful suggestions.

\section{REFERENCES}

1. K. Goebel and W. A. Kirk, A fixed point theorem for transformations whose iterates have uniform Lipschitz constant. Studia Math. 47(1973), $135-140$.

2. K. Goebel, W. A. Kirk, and R. L. Thele, Uniformly Lipschitzian families of transformations in Banach spaces. Can. J. Math. 26(1974), $1245-1256$. 
3. H. Ishihara, Fixed point theorems for Lipschitzian semigroups. Canad. Math. Bull. 32(1989), 90-97.

4. A. T. Lau, Semigroups of nonexpansive mappings on a Hilbert space. J. Math. Anal. Appl. 105(1985), 514-522.

5. T. C. Lim, Fixed point theorems for uniformly Lipschitzian mappings in $L^{p}$ spaces. Nonlinear Analysis 7(1983), 555-563.

6. T. C. Lim, H. K. Xu, and Z.B. Xu, Some $L^{p}$ inequalities and their applications to fixed point theory and approximation theory. Progress in Approximation Theory (P. Nevai and A. Pinkus, Eds.), Academic Press, New York, 1991, 609-624.

7. T. Mitchell, Topological semigroups and fixed points. Illinois J. Math. 14(1970), 630-641.

8. N. Mizoguchi and W. Takahashi, On the existence of fixed points and ergodic retractions for Lipschitzian semigroups in Hilbert spaces. Nonlinear Analysis 14(1990), 69-80.

9. W. Takahashi, Fixed point theorem and nonlinear ergodic theorem for nonexpansive semigroups without convexity. Can. J. Math. 44(1992), $880-887$.

10. K. K. Tan and H. K. Xu, Fixed point theorems for Lipschitzian semigroups in Banach spaces. Nonlinear Analysis 20(1993), 395-404.

11. H. K. Xu, Fixed point theorems for uniformly Lipschitzian semigroups in uniformly convex spaces. J. Math. Anal. Appl. 152(1990), 391-398.

12. H. K. Xu, Inequalities in Banach spaces with applications. Nonlinear Analysis 16(1991), 1127-1138.

(Received 15.07.1994)

Authors' addresses:

Kok-Keong Tan

Department of Mathematics,

Statistics and Computing Science

Dalhousie University

Halifax, Nova Scotia

Canada B3H 3J5

Hong-Kun Xu

Institute of Applied Mathematics,

East China University of Science and Technology,

Shanghai 200237,

China 